\title{
Cristallisation de l'image du Turkestan russe dans les premières expositions « coloniales » en Russie
}

\section{Svetlana Gorshenina}

\section{OpenEdition}

1 Journals

Édition électronique

URL : http://journals.openedition.org/edl/378

DOI : 10.4000/edl.378

ISSN : 2296-5084

Éditeur

Université de Lausanne

\section{Édition imprimée}

Date de publication : 15 septembre 2009

Pagination : 69-84

ISBN : 978-2-940331-20-8

ISSN : 0014-2026

Référence électronique

Svetlana Gorshenina, «Cristallisation de l'image du Turkestan russe dans les premières expositions " coloniales » en Russie », Études de lettres [En ligne], 2-3 | 2009, mis en ligne le 15 septembre 2012, consulté le 18 décembre 2020. URL : http://journals.openedition.org/edl/378 ; DOI : https://doi.org/ 10.4000/edl.378 


\section{CRISTALLISATION DE L'IMAGE DU TURKESTAN RUSSE DANS LES PREMIÈRES EXPOSITIONS "COLONIALES" EN RUSSIE ${ }^{1}$}

L'article propose une comparaison entre deux scénarios représentatifs de la construction de l'image du Turkestan russe à l'occasion des expositions «coloniales» russes, celui d'un peintre, Vassili Verechtchaguine, et celui d'un savant, Alexeï Fedtchenko. Malgré certaines différences de conceptions, nous montrons que ces expositions constituent des étapes logiques dans l'élaboration de la représentation exotisante de l'Asie centrale en tant que colonie russe et sont parfaitement conformes aux ambitions de leur commanditaire, Konstantin von Kaufmann, premier Gouverneur-Général du Turkestan.

Dans les analyses des expositions coloniales le Turkestan russe fait figure de grand absent. A la fin d'une abondante lecture, on peut parvenir à l'idée que ce "cœur du continent» n'a sous cet angle quasiment jamais été traité «à la une» des presses internationales de l'époque. Si le public occidental a bien été fasciné par les collections privées rapportées du Turkestan, on ignore en revanche que la Russie avait chez elle aussi consacré à cette région un certain nombre d'expositions de caractère colonial. Le problème qui se pose aujourd'hui est de savoir si le système des images des «indigènes» du Turkestan, propagé par la Russie à travers des expositions nationales et internationales, a produit un mécanisme de représentation de l'altérité «à l'européenne» ou "spécifiquement russe». En entraînant le Turkestan russe sur le terrain aujourd'hui bien

I. Le présent article est un extrait remanié d'un texte plus détaillé qui sera publié prochainement sous le titre "La construction d'une image "savante" du Turkestan russe lors des premières expositions "coloniales" dans l'Empire: analyse d'une technologie culturelle du pouvoir». 
apprivoisé des études sur les représentations coloniales, cette étude tentera d'analyser les étapes initiales de la construction de l'image de l'Asie centrale en tant qu'espace colonisé. Afin de mettre en relief les particularités et les traits communs qui inscrivent le Turkestan dans les phénomènes plus globaux, on s'arrêtera ici sur deux expositions représentatives : celle que le peintre Vassili V. Verechtchaguine conçut à SaintPétersbourg en 1869 et celle que le naturaliste Alexeï P. Fedtchenko monta à Moscou en 1872. On observera comment dans ces deux cas de figures le premier Général-Gouverneur du Turkestan Konstantin K. von Kaufmann s'est présenté comme le personnage clef qui aura dicté à lui seul toute la stratégie de construction des représentations. Conscient de l'impact que devait pouvoir produire l'image médiatique du Turkestan russe, cette importante figure de la colonisation russe s'est en effet tout particulièrement attachée à la recherche d'une formule visuelle susceptible de traduire le particularisme [unikal'nost'] du Turkestan.

\section{Le Turkestan de Verechtchaguine. L'exposition de 1869 à Saint-} Pétersbourg sur les productions de la région [Vystavka proizvedenij kraja]: une image incomplète, ambiguë et trop cruelle du Turkestan

Lorsque Kaufmann se lance dans la conquête du Turkestan, il prend soin de s'adjoindre des spécialistes de toutes les disciplines, à l'exemple de Napoléon Bonaparte qui avait entrepris sa campagne d'Egypte en compagnie d'artistes et de savants. Le peintre Vassili V. Verechtchaguine (1842-1904) se présente comme l'un des accompagnateurs les plus enthousiastes qu'excite tout particulièrement l'idée de participer à une «vraie guerre». Entré au service de Kaufmann dès le mois d'août 1867, le peintre s'active jusqu'à ce que, terrassé de fatigue, il se retire pendant l'hiver 1868-1869 à Paris où il va mettre ses observations au point. Soucieux de présenter ses œuvres "turkestanaises" après son retour à Saint-Pétersbourg, il propose alors à Kaufmann de mettre sur pied une exposition sur le sujet.

Cette initiative tombe au bon moment, car Kaufmann subit alors de violentes critiques et, pour justifier sa raison d'être, cherche à prouver la rentabilité du Turkestan. En Russie, cette nouvelle colonie n'est en effet pas perçue comme un «diamant précieux». Des spéculations contradictoires provoquent des éclats dans la presse et dans les hautes sphères 
politiques de la Russie. D’un côté, on souligne la haute importance stratégique et politique que revêt le Turkestan de par sa position centrale, avant d'évoquer le potentiel économique que cette région pourrait présenter tant pour le commerce que pour l'industrie russes, sans oublier tout un discours idéologique qui, au même endroit, situe la proto-patrie des Aryens. D'un autre côté, on souligne les difficultés d'une colonisation dans un climat hostile, la non-rentabilité des nouvelles possessions et la menace d'un affaiblissement de la Russie. Il faudrait alors, selon Kaufmann, effectuer auprès de l'opinion publique une valorisation de la colonie turkestanaise et construire une image positive au sein de la société russe.

A cette fin, l'enthousiasme du peintre se conjugue aux relations personnelles de Kaufmann et à sa caisse noire. Sans élaborer aucun programme précis et avec l'unique appui des objets de collections privées, Verechtchaguine monte en un temps record une exposition gratuite au Palais du Ministère des biens nationaux. Ce projet s'appuie sur la présentation de "créations artisanales", de collections naturelles, d'une collection archéologique et de trophées militaires célébrant vigoureusement la gloire de l'armée russe. Toutefois la salle où les foules vont se bousculer est surtout celle où Verechtchaguine présente ses œuvres.

La réaction du public, relayée par la presse, est teintée de perplexité. D’une part, le topos, selon lequel tout dans la région doit éclater de richesse "asiatique» - tout le monde a lu les Mille et une nuits! -, revient sans cesse en avant. D'autre part, la presse parle du "quotidien sans prétention du Centre-Asiatique», de la qualité médiocre des objets et de la situation défavorisée de la femme en Orient. Ces jugements de valeurs fleurissent à merveille dans le cadre des discussions très animées que suscitent les tableaux de Verechtchaguine. Accompagnées des notes de voyage que le peintre publie au cours de l'exposition dans le journal Golos [La Voix] en se présentant comme observateur attentif des événements in situ, ces œuvres constituent dans l'esprit du public un calque parfait de la nouvelle colonie russe. Chaque personnage de Verechtchaguine est vu comme un personnage type de l'Asie centrale qui doit incarner une "race». Mais la typologie physionomiste demande également des précisions sur la question qui brûle toutes les lèvres: comment ces "peuplades" se comportent-elles à l'égard des Russes? L'image des "indigènes" dans les croquis de voyages semble trop positive. Contrastant avec les dessins au crayon, les grands tableaux 
apportent une autre nuance dans la perception de la société «indigène». Dans des scènes qui se veulent "également très typiques", les caractères et le partage des rôles ne laissent planer aucune ambiguïté chez les critiques russes. Certains tableaux reflètent une barbarie des mœurs quasi palpable, comme dans le cas des Adorateurs de batcha [Obožateli bači], où de vieux et riches Sartes, emportés par des sentiments charnels, déshabillent d'un regard voluptueux un jeune garçon. Un même constat de décadence frappe «l'esprit civilisé» des commentateurs dans le tableau des Amateurs d'opium [Ljubiteli opiuma/Opiomoedy].

Ces sentiments de peur latente, de dégoût et de paternalisme se renforcent au travers d'autres sujets. C'est, en effet, en recourant à des expressions semblables que - à travers la symbolique simpliste d'une opposition entre une civilisation qui maîtrise froidement ses facultés et une barbarie indécente - la presse émet un verdict sévère à l'égard de deux tableaux liés à la dernière guerre avec Boukhara. Succès [Udača] et Echec [Neudača] sont perçus comme des œuvres complémentaires qui, en adoptant le point de vue de l'ennemi, racontent respectivement une victoire et une défaite des Turkestanais. Le premier tableau illustre une scène où, se laissant aller à un "rire indécent», les Turkestanais recueillent dans des sacs les têtes coupées de soldats russes dans le but de recevoir une récompense de l'émir. Pour illustrer un échec des «indigènes" le second tableau montre un soldat russe vêtu d'un uniforme en train de fumer sa pipe, la conscience tranquille, à proximité de cadavres d'ennemis tués lors de l'assaut de Samarkand. Selon la presse, on voit bien à travers ces images quel «type de vie les conquérants [russes] du Turkestan ont été contraints de mener en un premier temps» dans ce "pays de barbarie cruelle». Verechtchaguine tente cependant de nuancer cette image trop contrastée, en disant que dans cette guerre en Asie centrale "les adversaires ne se respectent pas réciproquement ${ }^{2}$. Ces observations passent pratiquement inaperçues et ces images, maintes fois publiées, deviennent le symbole d'une guerre avant tout engagée contre des barbares.

Dans tous les cas, il est clair aux yeux de l'opinion publique que «la grande masse du peuple [du Turkestan] est absolument étrangère au

2. Anonyme [1], «O dvuh kartinah na Turkestanskoj vystavke, zametka Vasilija Vereščagina", p. 23-25. 
peuple russe, et que, en plus, elle est à demi sauvage " ${ }^{3}$. Ce n'est pas un hasard si l'un des commentateurs se focalise tout particulièrement sur la description du milan et de l'aigle exposés comme en plein vol et qu'il définit comme des «représentants dignes de ces steppes, patrie de ces hordes carnassières" " ${ }^{4}$. Dans le même sens, des récits assez vagues circulent sur Severcev et Tatarinov qui, récemment libérés des prisons de l'émir de Boukhara, sont venus eux aussi présenter leurs collections naturalistes.

Kaufmann est assez satisfait des résultats. Premièrement, il reçoit des remerciements de la part du tsar Alexandre II, l'un des premiers visiteurs de l'exposition, qui par ce geste lui pardonne la conquête non autorisée de Samarkand. Deuxièmement, la presse s'est rangée à ses côtés. Cependant, une critique dérangeante parvient à le toucher, car, en précisant que l'exposition a été montée dans la précipitation, un auteur anonyme demande publiquement dans quelle mesure elle peut donner une image authentique de la nouvelle colonie ${ }^{5}$.

Les tableaux de Verechtchaguine, quant à eux, laissent flotter dans l'air le doute selon lequel l'image présentée du Turkestan ne serait pas suffisamment virile, car «les tableaux ne peuvent en aucun cas remplacer des mannequins ou, à la limite, des figurines regroupés selon une classification strictement scientifique ${ }^{6}$. Le public "éclairé» de SaintPétersbourg manifeste une intérêt plus net pour un tableau de profils ethnographiques construits selon des critères "vraiment scientifiques" fondés sur une classification de données taxonomiques précises comme la forme du crâne, puis la couleur des cheveux, de la peau et de l'iris.

En outre, trop cruelle, l'image du Turkestan de Verechtchaguine ne traduit pas suffisamment les efforts "constructifs" de l'administration locale dont le but est de montrer toute la séduction du nouveau territoire et son potentiel économique. Un nouveau défi s'offre au GénéralGouverneur: monter une exposition holiste du Turkestan, avec l'aide de scientifiques, pour contrecarrer toute critique susceptible de lui adresser le reproche d'une image partielle et faussée.

3. Anonyme [3], «O Turkestanskoj vystavke 1869 g.», p. 1-9.

4. Ibid.

5. M. Terent'ev, «Turkestanskaja vystavka», p. 59-71; Anonyme [2], «Estestvennoistoričeskij otdel na Turkestanskoj vystavke», p. 38-46.

6. Anonyme [3], «O Turkestanskoj vystavke 1869 g.». 
II. Le Turkestan de Fedtchenko. L'Exposition Polytechnique de 1872 à Moscou: une "vraie» image scientifique de l'Asie centrale

1. La rencontre décisive d'un Général-Gouverneur et d'un naturaliste

Dès le 30 décembre 1868 Kaufmann prend sous sa responsabilité l'accueil du naturaliste Alexeï P. Fedtchenko (1844-1873) qui, en 18691870, dirige l'Expédition scientifique turkestanaise, avant de proposer une présentation de l'Asie centrale russe à travers ses travaux dans le cadre de l'Exposition Polytechnique que l'OLEAE (la Société des amateurs d'anthropologie, d'ethnographie et des sciences naturelles) organise à Moscou, en 1872, comme un événement à l'échelle européenne afin de célébrer le $200^{\mathrm{e}}$ anniversaire de Pierre le Grand.

Cette occasion répond parfaitement aux objectifs de Kaufmann. Même si le faux testament du tsar cause bien des problèmes aux politiques russes, l'idée de présenter le Turkestan comme l'une des réalisations de ses projets semble très séduisante. Par la présentation de la région dans un pavillon séparé, cette exposition souligne la ressemblance de Tachkent avec Saint-Pétersbourg et, par sous-entendu, celle de Kaufmann avec Pierre le Grand. L'intérêt est d'autant plus grand que Kaufmann peut enfin confier la préparation de l'exposition à un "vrai " scientifique. Pour donner plus de poids au projet, le général et le scientifique décident en décembre 1870 de fonder un Département turkestanais de l'OLEAE dont Kaufmann devient le Président d'honneur. Cette alliance entre le scientifique et le Général-Gouverneur sera longue et fructueuse: pour le premier, libéral et lecteur des romans nihilistes de Nikolaï G. Tchernychevski, c'est un moyen d'obtenir les subventions nécessaires pour mener à bien et faire connaître ses travaux; pour le second, d'esprit conservateur, c'est une manière d'habiller les projets coloniaux d'un lexique scientifique.

2. L'élaboration du projet: une exposition holiste conçue selon des principes thématiques, géographiques et évolutionnistes

Partageant avec Kaufmann l'idée de l'unicité du Turkestan, le naturaliste écrit que les collections turkestanaises ne doivent pas embellir les autres départements de l'Exposition Polytechnique, mais être présentées dans un pavillon à part. Ce dernier doit être très décoratif pour 
mieux transmettre les particularités du pays: la solution qui semble la meilleure se présente sous la forme d'une copie de la somptueuse place du Reghistan de Samarkand avec ses trois madrasas. Ce décor doit coexister avec des imitations de montagnes, de steppes et de lieux d'habitations «indigènes", car, selon Fedtchenko, ce sont les trois paysages les plus significatifs de la région. Il doit également être complété par des plantes et des animaux vivants, ainsi que par des «indigènes, surtout des artisans, qui, en montrant leur art, devraient servir en même temps de représentants des types anthropologiques" ${ }^{7}$. L'exposition doit être bien structurée selon la pensée classificatoire occidentale, afin de donner un sens à la nature et de montrer son organisation sous-jacente: le Turkestan doit être présenté «dans les phases successives du développement historique et du travail technique pour montrer le progrès industriel et l'éducation publique offerts par la Russie" ${ }^{8}$.

Confiant en ses compétences, Kaufmann favorise toutes les démarches de Fedtchenko. En même temps, il apporte quelques modifications au plan établi. Tout d'abord il impose l'album photographique qu'Alexandre L. Kun (1840-1888) vient de terminer et qui, en offrant une vision totalisante du Turkestan en 1400 photographies, conclut l'un des plus importants projets d'archives coloniales lancés par Kaufmann. Puis, il introduit une Section militaire qu'il confie au général d'étatmajor V. N. Trotski, vice-président du Département turkestanais de l'OLEAE.

3. La préparation de l'exposition: changement de concept et coût d'une mise en scène "parfaite»

L'exposition étant conçue sur une très grande échelle, l'avancement des travaux va de pair avec l'accroissement des soucis financiers. Comme l'écrit Fedtchenko à Kaufmann:

[...] les chiffres des dépenses nécessaires pour construire les Sections m’ont fait réfléchir à propos du pavillon turkestanais. Bien que je ne sois personnellement pas intéressé à son existence, je suis convaincu

7. O. A. Maslova, Documentation réunie sur l'exposition de 1872 pour la biographie de O. A. Feď̌enko, 1. 40.

8. Ibid.; B. V. Lunin, Naučnye obščestva Turkestana i ih progressivnaja dejatel'nost'. Konec XIX-načalo XXv., p. 92. 
qu'il est une nécessité. A mon avis il serait préférable que les collections que j'ai réunies soient présentées dans le cadre des Départements zoologique et botanique de l'exposition. Mais au nom de l'intérêt du public et de l'administration du territoire il faudrait faire une présentation à part ${ }^{9}$.

La décision de relever le défi pour satisfaire les ambitions du GénéralGouverneur du Turkestan est d'autant plus significative que le Département turkestanais est le seul département régional. Dispersés dans soixante-dix pavillons, les vingt-quatre autres départements n'ont pas été conçus sur des principes régionaux, mais conformément à des spécialisations. Ce n'est que grâce à la volonté et au pouvoir de Kaufmann - qui débourse dans les 36000 roubles au lieu des 17000 prévus initialement -, que le Turkestan obtient un pavillon séparé, à la condition cependant que la présentation de ses collections soit mise en liaison directe avec l'OLEAE, donc, bien entendu, comme un exemple parfait des études scientifiques pluridisciplinaires relatives à un territoire.

\section{Un bijou oriental à l'ombre du Kremlin: visite guidée}

Dressé à l'ombre du Kremlin, le Département turkestanais domine le jardin Alexandre d'une façade qui, sur $33 \mathrm{~m}$ de haut, représente une imitation magnifique de la madrasa de Shir-Dor. A part la façade le seul détail reproduisant l'architecture traditionnelle de l'Asie centrale se situe à l'intérieur du pavillon sous la forme d'une cour et d'un jardin aménagés dans le "goût des Sartes" avec des galeries à colonnes «khiviennes" où fleurissent des plantes centre-asiatiques. Autour du jardin se succèdent des salles représentatives de l'image holiste du Turkestan: à gauche de l'entrée, le côté nord de la cour comprend plusieurs salles où sont exposées des collections d'histoire naturelle, de géographie et de statistique, puis une salle consacrée à l'agriculture que suit une salle dédiée à la technique; tout droit, vers le centre de l'ensemble, sont disposés des groupes ethnographiques; sur le côté sud, à droite, de petits espaces présentent une chambre «indigène", des collections militaires, des collections de Kouldja, ainsi que le bureau du Département ${ }^{10}$.

9. CGA RUz, f. I-1, op. 15, d. 96, 11. 87-87 verso.

Io. CGA RUz, f. I-1, op. 15, d. 127, 1. 4. 
Même si une partie des collections reste hors du pavillon, cartes, statistiques et graphiques de toutes natures couvrent de manière exhaustive apparemment tous les domaines. Au grand regret de Fedtchenko, ses collections de faune (qui regroupent 1584 spécimens ${ }^{11}$ ) sont en partie exposées au Musée de Zoologie, tandis que dans le pavillon on ne trouve que les exemples les plus spectaculaires d'animaux, d'oiseaux, d'insectes et de poissons, sous forme de spécimens empaillés, conservés dans l'alcool, séchés ou limités aux squelettes ou aux crânes. Les animaux vivants - qui comptent entre autres dix yaks tibétains - sont exposés au zoo de Moscou en raison de problèmes de place et d'entretien ${ }^{12}$. En revanche, les plantes vivantes cultivées au jardin botanique de l'université de Moscou côtoient de riches herbiers et des plantes conservées dans l'alcool; émaillées de spécimens neufs et inconnus, ces collections présentent la végétation du désert, des steppes, des bords de rivières, des oasis et des montagnes. Les échantillons minéralogiques et les fossiles constituent de riches collections qu'accompagne une carte stratigraphique des mines du Turkestan.

Ce panorama naturaliste organisé selon les principes positivistes au goût de Linné s'impose également dans la Section anthropologique. Pour illustrer l'évolution des "races" qui, selon Darwin, suppose un déterminisme biologique de la culture, quelques crânes sont exposés à côté de cerveaux conservés dans l'alcool, de têtes momifiées et de squelettes. Destinés à décrire les sédentaires de type "yagnob» du haut Zerafshan, ceux de type "tadjik" de Boukhara ou ceux de type "ouzbek» de la vallée du Zerafshan, de Samarkand et de Tachkent, ces témoignages sont combinés aux photographies des "types anthropologiques» réalisées par A. L. Kun ${ }^{13}$. A la différence du projet initial de Fedtchenko cette salle ne comprend cependant pas de «représentants vivants».

La Section d'agriculture aligne plantes agricoles, semences, outils agricoles «indigènes», ainsi que des échantillons représentant des terres diverses.

II. B. V. Lunin, Naučnye obščestva Turkestana i ih progressivnaja dejatel'nost'. Konec $X I X$-načalo $X X$ v., p. 93.

I2. CGA RUz, f. I-1, op. 15, d. 96, 11. 74-75, 113.

I3. O. A. Maslova, Documentation réunie sur l'exposition de 1872 pour la biographie de O. A. Fedčenko, 11.9 verso-14. 
La Section technique présente un caractère avant tout futuriste: en portant l'accent sur l'industrie du coton et de la soie, elle déroule les perspectives de développement de toutes les branches productives, aussi bien dans les domaines de l'agriculture, de l'horticulture et de l'élevage, que dans ceux de l'industrie lourde et légère. On y fait travailler des artisans-«indigènes» censés illustrer la "productivité locale» ${ }^{14}$.

La Section ethnographique vise à coordonner plusieurs niveaux de représentations. Jugée par les visiteurs comme la plus intéressante, elle occupe une surface plus grande que les autres: dans la cour intérieure du pavillon on peut admirer des modèles de bateaux, des exemples d'habitations de nomades et de sédentaires riches et pauvres, ou des bâtiments publics et religieux, ainsi que des instruments de musique et des jouets; trois tentes de types différents et une kibitka de Kirghizes se dressent à l'extérieur du Département; sur la place ouverte à l'avant de ce dernier se dresse une imitation du bazar de la place du Reghistan à Samarkand avec son labyrinthe de ruelles. Cette Asie apprivoisée est peuplée de quarante-quatre mannequins de Turkestanais.

Le public est enchanté par le "réalisme» des scènes, mais complète sa vision à travers les commentaires de la presse: malgré les couleurs enchanteresses il faut voir le bazar comme un endroit malpropre, mal aéré, habituellement délaissé à l'exception des jours de marché; en même temps, insistent les journalistes, c'est au marché que se concentre la vie sociale des Sartes qui s'y rendent pour discuter des nouvelles, regarder un balagan ou écouter les divana, sans dépenser trop d'argent, car dans le caractère des Sartes ce sont l'avarice et le goût du petit commerce de détail qui l'emportent; dans la maison de thé les Sartes se parlent paresseusement, fument le chilim [narghilé] et ne boivent que du thé vert, que l'on apprécie le plus en raison de son goût et de son effet narcotique ${ }^{15}$. "Passez plus loin, invitent les journaux: dans une boutique sous vos yeux le coiffeur rase la tête d'un Sarte, mais l'outil qu'il utilise inspire l'effroi, car il ne ressemble pas à un rasoir... ${ }^{16}$. La vie quotidienne des Kirghizes inspire tout autant de remarques. L'exposition d'une scène

I4. CGA RUz, f. I-1, op. 15, d. 127, 11. 12, 16, 29; Anonyme [4], «Moskovskaja Politehničeskaja Vystavka. Turkestanskij otdel», p. 244-245.

I5. O. A. Maslova, Documentation réunie sur l'exposition 1872 pour la biographie de O. A. Fedčenko, 11. 29-33.

I6. Anonyme [5], "Turkestanskaja vystavka», p. 235-237. 
de danse de batcha, allusion évidente au tableau de Verechtchaguine, celle des drogues consommées par les «indigènes » à côté de leurs boissons préférées, ou celle des épices et plats traditionnels, font défaut (censure?) dans la version finale, malgré leur présence à des étapes avancées du projet. Dans ce contexte le message implicite suggéré par le bâtiment à forme de Shir-Dor souligne davantage le caractère primitif du monument que sa splendeur. La toile remplaçant les faïences ne transmet que le caractère "trop chargé» de l'ornementation de cette architecture orientale. Cette mise en scène ne laisse percevoir la société traditionnelle turkestanaise que sous son aspect purement matériel: la seule allusion à la vie religieuse (la façade de Shir-Dor n’a pas été décrite comme appartenant à un bâtiment religieux) se fait à travers les figures des divana, dont la présence accentue les différences culturelles, réaffirmant ainsi l'«altérité» des Turkestanais.

Pour finir, à côté du Département militaire, près de l'entrée du pavillon, se dresse une petite librairie destinée à la diffusion des publications réalisées à l'occasion de l'exposition, qui comprennent notamment un catalogue raisonné, ainsi que trois volumes du Turkestan russe qui font également connaître la «vraie physionomie» du Turkestan. Le principe de l'exhaustivité des connaissances, qui s'était déjà fortement exprimé en 1867 à l'Exposition universelle de Paris ${ }^{17}$, trouve donc sa manifestation la plus parfaite dans le pavillon du Général-Gouverneur.

5. Le démontage du mythe d'une colonie pleine de défauts: un triomphe de l'image holiste et scientifique

Les résultats de l'exposition sont au niveau des attentes et des dépenses. Le Département turkestanais est souvent comparé par le public au Département de la marine, clou de cette même Exposition Polytechnique consacrée à Pierre le Grand, et récolte une riche moisson de récompenses en médailles et en titres. La presse russe est unanime dans ses conclusions: «le Département turkestanais dépasse largement bien d'autres départements de l'Exposition Polytechnique » ${ }^{18}$. Avec cette

17. P. Greenhalgh, Ephemeral vistas: the "expositions universelles", great exhibitions and world's fairs, 1851-1939, p. 20.

I8. Zemledel'českaja gazeta; O. A. Maslova, Documentation réunie sur l'exposition de 1872 pour la biographie de O. A. Feď́enko, 1. 106. 
haute estimation du Département la valeur du Turkestan est pleinement reconnue en tant qu'aire géographique et comme tout à fait comparable aux pays de l'Orient classique tels que l'Inde, la Chine ou l'Egypte: après le constat des richesses qui y sont présentes, chaque compte rendu finit par brosser à grands traits les perspectives de développement du Turkestan. En même temps, un discours sur la nécessité d'une immigration soutenue se manifeste de plus en plus clairement. Le rôle que doit jouer l'administration, évidemment éclairée et soucieuse de développer le pays, n'est jamais oublié.

En effet, Kaufmann parvient à se faire pardonner ses conquêtes non autorisées et obtient un statut particulier pour pouvoir assurer une immigration régulière; il se voit alors accorder carte blanche pour lancer des actions expansionnistes aux dépens des territoires adjacents que l'on considère comme extrêmement utiles pour la Russie.

Le résultat majeur de cette exposition concerne surtout l'image construite par Kaufmann. Créée avec l'appui de Fedtchenko, cette image est reconnue par les grands savants comme "authentiquement scientifique" au point que le contenu de l'exposition est acquis par le nouveau Musée Polytechnique grâce, une fois de plus, à un legs de Kaufmann ${ }^{19}$. L'image d'une colonie riche et rentable qui, grâce à des Russes débrouillards, se lance sur la voie de la "civilisation" revêt un lustre scientifique tout à fait à la hauteur des autres puissances coloniales.

III. Les "particularités russes" des expositions coloniales du Turkestan: une présentation de "semblables» et l'impérialisme "privé» de Kaufmann

La présente reconstitution des premières étapes de l'élaboration de l'image du Turkestan à travers les expositions nationales montre comment la réalité d'une technologie culturelle du pouvoir s'érige dans une imbrication complexe de connaissances coloniales, d'arts et de pouvoirs coloniaux.

La nature de cette symbiose ne permet pas, semble-t-il, de différencier la Russie des autres puissances européennes coloniales. En récupérant le schéma des expositions coloniales britanniques ou françaises et avec l'appui d'artistes et de scientifiques, ainsi que de militaires éduqués

19. CGA RUz, f. I-1, op. 15, d. 127, 11. 3-29. 
et souvent employés pour des recherches, l'administration coloniale turkestanaise construit ses représentations dans l'axe des mêmes idées clefs: la transmission du Progrès et de la Modernisation à des peuplades arriérées.

Les conséquences de cette imbrication sont également comparables dans le monde occidental: l'appropriation de l'espace conquis et sa transformation en territoire apprivoisé sont toujours au centre de n'importe quelle entreprise coloniale. Cette politique s'exprime par un droit d'inventaire total: tout enregistrer, cartographier, classifier, catégoriser, délimiter avant de tout soumettre à ses ordres pour le transformer conformément aux schémas impérialistes. La création de collections et d'archives coloniales, ainsi que leur exposition dans le cadre d'une mise en scène voulue, supposent l'introduction de nouvelles catégories politiques, scientifiques et culturelles élaborées par le pouvoir et pour le pouvoir.

Les «spécificités russes" sont plutôt ailleurs. Premièrement, dans le discours identitaire ambigu que, depuis l'époque de Pierre le Grand et, surtout, dès les années 1820 les intellectuels russes tiennent avec force en déclarant la Russie tantôt européenne, tantôt asiatique, la présentation de l'Asie ne peut être exactement la même qu'en Occident. Le scénario des "villages nègres" se révèle inutilisable pour intégrer les Turkestanais au discours impérial russe, même si des "objets vivants" émaillent les expositions russes. En prétendant être en même temps aryens et touraniens, les commissaires russes présentent dans les expositions turkestanaises des individus «semblables» plutôt qu' «exotiques», en "exotisant» toutefois l'image générale du Turkestan.

L'Autre n'incarne pas vraiment une altérité raciale, mais plutôt une altérité culturelle et morale. La couleur de la peau, marqueur emblématique de la différence, n'est pas pertinente par rapport aux Turkestanais; ce taxome selon les schémas de l'époque s'étale dans ce cas sur des nuances graduelles entre Indo-Européens, sans jamais associer les Centre-Asiatiques aux Noirs ou aux Jaunes.

Comparables aux expositions missionnaires en Italie où l'objectif éducatif l'emporte sur toute autre considération, les expositions turkestanaises prohibent toute suggestion traumatique ou morbide concernant les «indigènes» et privilégient des représentations «normalisées» avec un accent fort sur la russification [obrusenie] et une assimilation possible des populations asiatiques, en montrant le rapprochement progressif des 
cultures et des modes de vie des «indigènes" vers le modèle russe. Cela permet d'échapper au «bric-à-brac exotique» des premières expositions coloniales européennes, en privilégiant dès le début un aspect "sérieux" de l'approche, même trop sérieux, devrait-on dire, car ni la danse, ni la musique, ni l'exécution de rites traditionnels ne sont requis par les premiers commissaires russes.

Les expositions coloniales turkestanaises présentent encore une autre particularité: l'impérialisme "privé» de Kaufmann. Au lieu d'être le produit d'une propagande de l'Etat ou le fruit d'une coopération entre l'Etat, des entrepreneurs privés, des villes ou des gouverneurs coloniaux, comme cela a été le cas en Europe ${ }^{20}$, les manifestations russes sont des créations quasi personnelles de Kaufmann. Il est d'ailleurs difficile d'imaginer un autre scénario dans ce contexte de politique non conséquente et hésitante de la Russie en Asie centrale où les promesses internationales ne sont jamais tenues et les initiatives privées des militaires sont punissables en cas d'échec, mais toujours bien acceptées en cas de victoire.

En outre, les expositions coloniales ne sont pas seulement l'occasion de présenter un monde colonial et étranger, mais aussi celle d'étaler sa grandeur nationale. La présentation du Turkestan dans des expositions nationales permet à la Russie de se récompenser après la défaite de la guerre de Crimée de 1853-1856 par l'expression visible et triomphante de la suprématie de la civilisation russe tant à l'égard de l'Asie que par rapport à l'Europe. Comme les autres puissances européennes, la Russie les met à profit pour valoriser son particularisme dans la construction de sa propre image, en exhibant ses projets sociaux et sa vision du monde.

Svetlana Gorshenina

Paris-Lausanne

20. P. Greenhalgh, Ephemeral vistas: the "expositions universelles", great exhibitions and world's fairs, 1851-1939, p. 28-41. 


\section{BIBLIOGRAPHIE}

\section{Abréviations}

CGA RUz: Central'nyj gosudarstvennyj arhiv respubliki Uzbekistan / O'zbek Respublikasi Markaziy Davlat Arhivi [Archives centrales d'Etat de la République d'Ouzbékistan].

OLEAE: Obščestvo ljubitelej estestvoznanija, antropologii i etnografii [Société des amateurs d'histoire naturelle, d'anthropologie et d'ethnographie].

Archives

CGA RUz: fond I-1 (Kanceljarija Turkestanskogo General-Gubernatora [Chancellerie du Général-Gouverneur du Turkestan], opis' 15, dela 96, 127.

\section{Textes et études}

Anonyme [1], "O dvuh kartinah na Turkestanskoj vystavke, zametka Vasilija Vereščagina", Sankt-Peterburgskie vedomosti, 1869, in Turkestanskij sbornik, t. XXVII, 1870, p. 23-25.

Anonyme [2], «Estestvenno-istoričeskij otdel na Turkestanskoj vystavke", Vsemirnaja illjustracija, 19-21, 1869, in Turkestanskij sbornik, t. XXVII, 1870, p. 38-46.

Anonyme [3], "O Turkestanskoj vystavke 1869 g. ", Vestnik Evropy, 1869, $\mathrm{n}^{\circ}$ 5, p. 373-380, in Turkestanskij sbornik, t. XXVII, 1870, p. 1-9. 
Anonyme [4], «Moskovskaja Politehničeskaja Vystavka. Turkestanskij otdel ", Moskovskie vedomosti, 110, 1872, in Turkestanskij sbornik, t. XLIII, 1873, p. 244-245.

Anonyme [5], "Turkestanskaja vystavka», Vsemirnaja illjustracija, in Turkestanskij sbornik, t. XLIII, 1873, p. 235-237.

Gorshenina, S., "La Construction d'une image “savante” du Turkestan russe lors des premières expositions "coloniales" dans l'empire: analyse d'une technologie culturelle du pouvoir ", in S. Gorshenina et S. Abashin (éds), Turkestan russe: une colonie pas comme les autres? (Collection de l'IFEAC - Cahiers de l'Asie centrale), Paris, Complexes, 2009 (sous presse).

Greenhalgh, P., Ephemeral vistas: the "expositions universelles", great exhibitions and world's fairs, 1851-1939, (Collection Studies in imperialism XII), Manchester, Manchester Univ. Press, 1994.

Lunin, B. V., Naučnye obščestva Turkestana i ih progressivnaja dejatel'nost'. Konec XIX-načalo XX v., Taškent, Izdatel'stvo Akademii nauk UzSSR, 1962.

Maslova, O. A., Documentation réunie sur l'exposition de 1872 pour la biographie de O. A. Fedčenko, s/d, in CGA RUz, f. R-2691, i. 1, d. 10.

Terent'ev, M., "Turkestanskaja vystavka», Russkij invalid, 45, 1869, in Turkestanskij sbornik, t. XXVII, 1870, p. 59-71.

Zemledel'českaja gazeta, 38, 1872. 